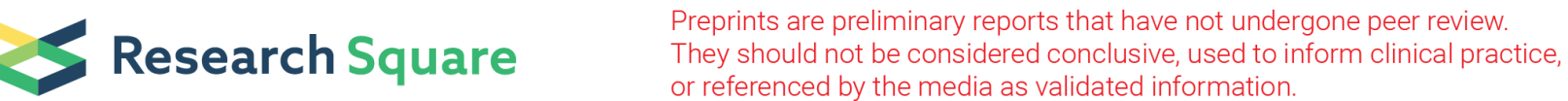

\section{Does The Taste of The Plain Packaged Cigarettes in Saudi Arabia Really Changed and Differ from The Branded Cigarettes or it is A Natural Sensory Change? Experimental Study}

Nasser F Bin Dhim

sharik Association for health research

Mada H Basyouni

Ministry of Health

Nora A Althumiri ( $\nabla$ nora@althumiri.net)

Sharik Association For Health Research https://orcid.org/0000-0002-8215-6097

Rasha A. Almubarak

Saudi Food and Drug Authority

Weam Banjar

Ministry of Health

Mohammed Zamakhshary

Alfaisal University

Khaled M. AlKattan

Alfaisal University

Research article

Keywords: Plain Packaged, Tobacco, Smoking Regulation, Public Policy

Posted Date: July 8th, 2020

DOI: https://doi.org/10.21203/rs.3.rs-32844/v1

License: (1) This work is licensed under a Creative Commons Attribution 4.0 International License.

Read Full License 


\section{Abstract}

Background: Does the taste of the plain packaged cigarettes recently implemented in Saudi Arabia really changed and differ from the branded cigarettes or it is a natural sensory

Methods: 18 adult smokers, who are currently smoking cigarettes and cleared upon physical assessment before the experiment. Participants were excluded if they were planning to quit smoking or in quitting stage or have any respiratory or cardiac disease, any taste related disorder (e.g. hypogeusia / ageusia) or any acute disease that affect taste or smell (e.g. Flu). Participants who shows any abnormal vital signs check before the study (fever, shortness of breath or elevated blood pressure). Participants received 6 sequences of different random exposures ( 3 puffs) to 3 plain packaged cigarettes ( 2 from the favorite brand and 1 other brand "control") and 3 branded cigarettes (2 from the favorite brand and 1 other brand "control"). Participants wore Virtual Reality Goggles (VR) accompany with a special software to alter the visual reality and wore gloves to alter the touch sensation.

Results: Participants were not able to identify the correct type of cigarettes (plain or branded (Estimate of fixed effect $-0.01 p=0.79$ ). Moreover, there were no differences in the ability of the participants to identify their favorite brand $t(-0.63)$ mean $=0.47 p=0.53$.

Conclusion: After controlling the visual and touch sensation participants were not able to difference between branded and plain packaged cigarettes in terms of taste or inducing immediate shortness of breath or cough. Interestingly, participants were not able to identify their favorite brand.

\section{Background}

The Prevalence of cigarette smoking among adults in Saudi Arabia is between $16.1 \%$ to $21.1 \%$. $(1,2)$ Saudi Arabia implemented tobacco plain packaging in August 2019. After few weeks of implementation huge number of smokers complained in various media channels especially social media about extreme change of how the cigarette taste, increase the frequency of coughing, and for some hospitalization caused by shortness of breath. The taste claims have been arise in other countries implemented plain packaging like Australia and United Kingdom.(3-5)

These complains persistently continued for more than 90 days, starting by the mid of November 2019 until the time of writing this draft $27^{\text {th }}$ Jan 2020 . Although chemical analysis found the plain packaged cigarettes meeting the Saudi standards set by the Saudi Food \& Drug Authority, rumors that the current cigarettes sold in Saudi Arabia in plain packaging have toxic chemicals or other non-tobacco substance intended to harm smokers are widely circulated in the media and common by word of mouth.

The Saudi Authorities requested tobacco companies to declare any changes of the cigarettes content outside the new plain packaging requirements. British American Tobacco Middle East (BAT) and Philip Morris International (PMI) released public statements declaring no changes to their products content (Appendix 1). 
Nevertheless, citizens and visitors to Saudi Arabia are anxious and concerned about the health consequences of consuming the current plain packaged cigarettes in Saudi Arabia. Consequently, prices of the branded cigarettes were tripled and smuggling dramatically increased.

The results of chemical analysis and conformity with Saudi Standard have showed that the new plain packaged cigarettes are within the standards, and no unusual toxicity has been found. However, confirmation of the claims made on the media channels about the taste, immediate coughing or shortness of breath have not been investigated.

There are few studies that have investigated this issue in other countries that implemented the plain packaging. (6-8) These studies did not find significant difference in taste, however, they highlighted the difficulties of measuring it that might affect the results.

\section{How Changing Vision and Touch Senses Affects Taste}

It is known that the stimulation of one sense organ influences in some extent the sensitivity of organs of another sense.(9) Human beings have five senses that are interlinked to each other. Changing or altering the vision or touch senses affects taste. In fact, taste involves the combination of gustatory and olfactory stimuli.(10) And despite the fact that vision is not directly related to taste, changing vision alters the perception that a person might have about something thus leading to a change in its taste.(11) When individual taste something that they are visually seeing, there are chances that the taste will change based on what the individual had registered in the brain. "A mouthful usually stimulates sight first and then touch, taste and smell".(12) However, there are possibilities that the vision might have a wrong interpretation of the taste. Therefore, changing vision affects the taste based on the person's perception. (13) Moir (1936), has published more than 150 studies examining vision's impact over taste and flavor. (14) The majority of this research has proven that changing the hue and/or intensity of the color added to a food or, a beverage can affect the perceived identity and/or intensity of the flavor.(14)

Apart from vision also altering touch also affects taste. The touch system contributes to this constancy as taste sensations appear to be localized by touch.(15) Touch, whether in the mouth or on the hand, has a far influence on perception of taste, quality, and satiety more than we realize.(10) The tip of the mouth is an area of high receptor density and the taste sensation begins to weak on the inside and gets stronger at the tip.(15) Thus, research for years has investigated how the act of touching products affects consumer response. Therefore, the firmness of a cup in which water is served may affect consumers' judgments of the water itself.(13)

\section{Effect of prominent Pictorial Warning}

The recently implemented tobacco plain packaging regulation in Saudi Arabia was accompanied with the implementation of new set of prominent pictorial warnings compared to the previously implemented ones. This change is the first in terms of changing pictorial warnings since its introduction in Saudi Arabia in August 2011.(16) 
Previous study in Saudi Arabia investigating the effect of prominent pictorial warnings on Saudi sample showed that the prominent pictorial warnings received on average 13.1 and 10.2 rating points on the Brief Worry Scale about Smoking (BWS) and on average 12.5 and 10.1 on Self-Assessment Manikin (SAM) compared to the old pictorial warning.(16) which indicate expected stronger emotional evocation to the change in pictorial warnings in Saudi Arabia.

Thus, this small trial will strive to address the following concerns in the most scientific and ethically possible ways:

1. Does the taste of the plain packaged cigarettes really changed and differ from the branded cigarettes or it is a natural sensory change?

2. Dose the plain packaged cigarettes cause more immediate coughing compared to the branded cigarettes?

3. Dose smokers who smoke plain packaged cigarettes suffer more shortness of Breath compared to the branded cigarettes?

In addition this article will discuss the lesson learned from the Saudi experience with regards to this trial findings and other available evidence.

\section{Methods}

\section{Study Design:}

One group crossover trial to answer the following question:

1. Does the taste of the plain packaged cigarettes really changed and differ from the branded cigarettes or it is a natural sensory change?

2. Dose the plain packaged cigarettes cause more immediate coughing compared to the branded cigarettes?

3. Dose smokers who smoke plain packaged cigarettes suffer more shortness of Breath compared to the branded cigarettes?

4. Qualitative interview after the experiment to understand the results impact on the participant.

\section{Exposure and Procedure:}

Participants received 6 sequences of different random exposures to 3 plain packaged cigarettes ( 2 from the favorite brand and 1 other brand "control") and 3 branded cigarettes ( 2 from the favorite brand and 1 other brand "control"). With a wash-out period of 5-10 mins between each cigarette. To ensure reproducibility all participants were invited to repeat the trial in another day.

The standard cigarette in Saudi Arabia is approximately 5.5 Centimeters long excluding the filter, thus, for harm reduction reasons the smoker will be exposed to approximately 3 puffs which represent around 2 
Centimeters. To ensure that the smoker does not exceed this amount an aluminum foil will be wrapped around the rest of the cigarette.

To ensure concealment, the cigarettes were provided in random order by a blinding handler who are not involved in data collection and/or data analysis.

To blind participants from recognizing the type of cigarettes, the participant wore Virtual Reality Goggles (VR) accompany with a special software to alter the visual reality of the smoker (Figure 1). In addition, the participants wore medical gloves to alter the hand touch felling of the cigarettes.

\section{Inclusion and exclusion criteria:}

Participants were smokers 18 years old and above, who are currently smoking cigarettes and cleared upon physical assessment before the experiment.

Participants who are planning to quit smoking or in quitting stage are not eligible to participate in this study to reduce harm and ethically not altering their quitting process or plan. Participants who have any respiratory or cardiac disease, any taste related disorder (e.g. hypogeusia / ageusia) or any acute disease that affect taste or smell (e.g. Flu). Participants who shows any abnormal vital signs check before the study (fever, shortness of breath or elevated blood pressure).

\section{Recruitments.}

Participants were invited form Sharik research participants database(17), which include around 6000 smokers via phone call and study information and consent were presented to them, if agreed eligibility conditions checked and if eligible, an appointment booked to come to the study place. Once arrived the researchers explained all the details of the study to the participants and written participant information sheet were provided. Participants who want to start the study were asked to sign the consent. Then their vital signs were checked.

\section{data collection and outcome measure:}

Data collection started with a quick interview survey including the age, gender, Fagerstrom Test for Nicotine Dependence, age start smoking, frequency of coughing last 2 weeks.

The main outcome measure for the taste will be via asking the participants to identify the type of cigarette they smoked (plain or brand) based on taste. In addition, asking the smoker to rate the taste of the cigarette on a scale from 1 to 7 ( $1=$ very bad taste and $7=$ very good taste) and the heat perception of the cigarette smoke (burning sensation) from 1 to 7 (1= acceptable and 7=unacceptable). Finally, the smoker will be asked if the cigarette is from his favorite brand or not).

The outcome measure for immediate coughing is an observation of any coughing event during the smoking or washout period for each cigarette. 
The outcome measure for shortness of breath will be measured via self-reporting and peak flow test. The participants will repeat the test before and after each cigarette for safety reasons, however, the main measure here is the first cigarette comparison between (plain and brand) as it was delivered in random order for each participants. The reason for comparing only based on the first cigarette is that second cigarettes maybe of another type which will introduce contamination, in addition, to the accumulative effect of each experiment. The shortness of breath is defined on the peak flow if the reading is $40 \%$ less than the baseline before starting the first cigarette as readings of $50 \%$ less than the baseline is defined as the signal for medical alert.(18)

After finalizing the experiment, the participants were asked about their perception about the taste \& health concerns claims circulated in the media about plain packaged tobacco to be compared to their initial opinion before the experiment.

\section{Time Schedule:}

1. Participants recruitment started early January 2020 via phone interview.

2. Each eligible participant were scheduled to visit the study facility to start the study which took around (60 mins)

3. Participants then were invited to the second session within a week of the first session.

\section{Sample size:}

Based on the smokers complains on media channel it seems that the different in taste between the plain and branded cigarette are large to medium. Thus, A single-factor, repeated measures design with a sample of 18 subjects, measured at 6 time points to produce (108 experiment), achieves $80 \%$ power to detect a contrast using a multivariate $T^{2}$ test at a 0.05 significance level at $0.45-0.35$ effect size. $(19,20)$ Sample size was calculated via PASS 2019, v19.0.3 using the above mentioned inputs.

Expected Results \& Benefits:

1. Inform the authority about the existence of difference in taste form a study on local subjects. As very few previous studies where done in other countries.

2. Inform the Authority of any immediate harm on smokers using the plain packaged cigarette and help provide an evidence to end to the claims regarding the plain packaged cigarettes, which is currently a country-wide issue.

\section{Data Analysis}

Descriptive statistics were used to describe the sample demographics, Mixed model analysis will be used to analyze repeated measures of taste related outcomes. T test will be used to analyze ability of participants to identify their favorite brand and T test were used to analyze the follow update due to the small sample size which prevented the use of the repeated measure mixed model analysis. 


\section{Ethical considerations:}

The study was performed in agreement with the Declaration of Helsinki. Ethical Approval were obtained from Alfaisal University (Institutional Review Board).

\section{Results}

\section{Demographics and Baseline}

25 participants were approached out of them 1 participant were excluded because he was in quitting stage, and 2 for having a cardiovascular disease. 5 participants were eligible but did not show up. The 18 participants included 1 female (5.5\%) and 17 males (94.4\%), mean age 28.9 (range $19-63$ ), Mean

nicotine dependency score 3.3 (range $2-5$ ), and mean number of cigarettes smoked per day is 18.3 (range $12-24)$. In the last 2 weeks, 16 out of 18 participants (88.9\%) never had any cough attack and 2 participants had it once or more.

In terms of participants opination about changes in the new plain packaged cigarettes taste and content 16 out of 18 participants (88.9\%) think there is a change compared to the old branded cigarettes. However, after the experiment all reported that they do not believe there was any differences between plain and branded packaging cigarette.

\section{Taste Change}

In terms of the ability of the participant to identify the correct type of cigarettes (plain or branded) mixed model analysis showed no significant differences Estimate of fixed effect $-0.01 p=0.79$.

In terms of taste and burning sensation ratings, there were no significant differences in the rating scores between the plain and the branded cigarettes. Estimate of fixed effect $-0.31 p=0.30$ for taste and Estimate of fixed effect $-0.25 p=0.42$ for burning sensation.

Finally, there were no differences in the ability of the participants to identify their favorite brand $t(-0.63)$ mean $=0.47 p=0.53$.

\section{Chough and Shortness of Breath}

In terms of immediate coughing out of the 108 experiments 1 episode of short coughing was observed and it was attributed to the branded cigarette not the plain packaged.

In terms of shortness of breath non on the participants in the 108 experiments reported shortness of breath. In addition, comparing the changes in peak flow reading between the first cigarette none of the participants had any shortness of breath. Finally, no cases of shortness of breath has been recorded overall after the full experiment for all participants. 
Only 4 participants (22.2\%) attended the second session. The 4 participants have finished in total 48 experiments in the two sessions. There were no differences between the participants performance in session 1 compared to session 2 in identifying the (branded or plain cigarettes) $t(-0.96)$ session 1 mean $=0.33$, session 2 mean $=0.21 p=0.34$. In terms of taste rating there were no significant difference $t(-0.27)$ session 1 mean=3.67, session 2 mean=3.50 $p=0.79$, and no significant difference in terms of burning sensation $t(-0.60)$ session 1 mean $=4.66$, session 2 mean $=4.29 p=0.55$. No coughing or shortness of breath were observed.

\section{Discussion}

\section{Study Findings Summary.}

This study investigated the claims of taste change, immediate continuous coughing and immediate shortness of breath. After controlling the vision and touch perception of the participants there were no significant differences in their ability to identify plain versus branded cigarettes and more surprisingly no significant differences in their ability to identify their favorite brand. These were also no alarming findings in terms of immediate cough or shortness of breath.

\section{Summary of the issue escalation}

It seems that the visual and touch feelings contributed to the smoker's perception of taste change and via massive social media circulation of potential changes in the cigarettes content that can cause more health damage these changes got linked and exaggerated. This also made worse by introducing the new potent emotionally evoking pictorial warnings at the same time which in previous study proved to signal large health concern in the same population.(16)

One more issue in the Saudi implementation process is the lack of public awareness campaign before the implementation. The smokers were shocked "one day" with the new cigarette packaging and emotionally evoking pictorial warnings without any previous knowledge which direct them to seek answers and create their own theories.

In addition, after the escalation of the issue the official authorities did explained lightly that the plain packaging is a new regulation, however, the explanation did only explain the outer packaging not the internal (paper and filter) changes neither the new pictorial warning. This left majority with unanswered questions and concerns that as some express it "the new black packaged cigarettes is harmful and fake (counterfeit)."

The claims included endless of theories including that the new cigarettes is made of "sawdust" which was denied by the Saudi Food and Drug Authority.(21) After not being able to generate any laboratory confirming evidence that there were changes in the content of the cigarettes by the official authorities in Saud Arabia "Tobacco companies have been told to ensure their products meet customer expectations on flavor and quality by a Saudi government committee investigating smokers' complaints" and "Companies 
and corporate agents were also directed to communicate with consumers directly through contact points dedicated to handling complaints".(22) Tobacco companies then released their statements (Appendix 1) declaring no changes in the cigarette content except what was required by the new regulation without giving any details of what the new regulation asked for, and the dilemma goes on and on. At the moment of writing this manuscript the Authorities is considering revising the plain packaging standards to allow tobacco companies to reintroduce the cigarettes with their original branded (paper and filter) look while keeping the external plain packaging, which will be a victory for tobacco companies.

\section{Reorganizing the chain of events}

The implementation of plain packaging in Saudi Arabia has multiple major chain of decisions that led to the current situation which almost ruined the implementation of an effective public health policy. The major factors ordered as:

1. Lack of pre-implementation awareness campaign which played a major role in convincing the consumers that the plain packaged cigarettes is counterfeit.

2. Introducing the new pictorial warning at the sometime, having a previous knowledge that it may cause consumer anxiety, with no pre-implementation awareness campaign too.

3. Lack of prior risk assessment of the second order effect of such implementation on stakeholders.

4. Lack of awareness about the plain packaging standards explaining why the externals and internals of the products has changed and delay in response to consumers concerns especially health-related ones.

5. Lack of scientifically sound evidence to explain the claims of taste changes and health concerns.

The major lesson and factor here is the lack of proper risk assessment before implementation "having the right perception of risk is the holy grail of decision making". The impact of the new changes was a life change for a smoker who has not been used to such strong public health actions. As some of this study described it "I woke up someday and find out that all cigarettes brands are the same look and feel, it must be counterfeit, there is no other explanation".

The implementation also underestimated the role of social media in spreading fears and rumors to counteract the implementation of the new regulation especially with the known history of how tobacco industries strive to undermine tobacco control regulations. $(23,24)$ The claims about the plain packaging including taste and quality has been raised in countries that implemented the pain packaging regulation such as Australia and the United Kingdom(5-7), and the assumption that it was predictable for such claims to be reused in counter policy campaigns was also missed.

Instead of being proactive with the consumers and engaging with them to explain the new regulation the authorities kept silence for very longtime, with few contradicting announcements that started by denying and changes in physical and chemical content, then confirming the changes in quality and taste and blaming tobacco companies. Tobacco companies in return stated no changes. Nevertheless, until the 
time of writing this manuscript the consumers do not know what was changed, how, and/or why. None of the statements by authorities or tobacco companies explained to the consumer why all cigarettes look the same (paper and filter), and why it has new emotionally provoking pictorial warnings.

Unfortunately, the same repelling effects (evoking smokers health concerns and removing the appeal and charm of brands) that was desire to encourage smokers to quit and prevent non-smokers from smoking, when introduced in the wrong order and rushed to the implementation caused unexpected second order effect that neutralized the policy and as some may call it the biggest media campaign for tobacco products in the Middle East. Reading thorough the millions of posts in social media in a trending topic for more than 90 days, implemented one important idea in favors of tobacco companies "smoking is harmful, but plain packaged tobacco is the real killer".

\section{Conclusion}

After controlling the visual and touch sensation participants were not able to difference between branded and plain packaged cigarettes in terms of taste or inducing immediate shortness of breath or cough. Interestingly, participants were not able to identify their favorite brand.

\section{Declarations}

Ethical approval was granted from Alfaisal University Institutional Review Board with approval ID number IRB-20013. All participant has signed the consent form approved by Alfaisal University Institutional Review Board to participate in this study.

\section{Strengths and limitations of this study}

- This study provided new dimension to the reason why some smokers find the taste of plain packaged cigarette differ form the branded one.

- This study used innovative approach to alter the visionary sensation via virtual reality.

- This study is limited by its quasi-experimental design.

- This study is limited by its small sample which was balancing the need for the study and harm reduction.

\section{Consent for publication}

Not applicable.

\section{Funding}

This research project has not received any funding from public or private entity. All the research efforts in this study was provided voluntarily from the research. 
The authors have no competing or conflict of interest to declare.

\section{Authors Contributions}

All authors provided major contribution to the design and execution of this study. NFD designed the study and drafted the manuscript, MHB, NAA, RA, administered and managed the study and data collection, MZ and KMA contributed to the study design and reviewed the manuscript. WB finalized and edited the manuscript for publication. All authors approved the final version for publication.

\section{Availability of data and material}

Data is available upon request from the Corresponding Author.

\section{Acknowledgments}

None

\section{References}

1. Algabbani AM, Almubark R, Althumiri N, Alqahtani A, BinDhim N. The Prevalence of Cigarette Smoking in Saudi Arabia in 2018. Food and Drug Regulatory Science Journal. 2018;1(1):1-.

2. Algabbani AM, Althumiri NA, Almarshad AM, BinDhim NF. National prevalence, perceptions, and determinants of tobacco consumption in Saudi Arabia. Food and Drug Regulatory Science Journal. 2019;2(2):1-.

3. Miller CL, Brownbill AL, Dono J, Ettridge K. Presenting a strong and united front to tobacco industry interference: a content analysis of Australian newspaper coverage of tobacco plain packaging 2008-2014. BMJ open. 2018;8(9):e023485.

4. Moodie C, Angus K, Mitchell D, Critchlow N. How tobacco companies in the United Kingdom prepared for, and responded to, standardised packaging of cigarettes and rolling tobacco. Tobacco control. 2018;27(e1):e85-e92.

5. Evans-Reeves KA, Hiscock R, Lauber K, Gilmore AB. Prospective longitudinal study of tobacco company adaptation to standardised packaging in the UK: identifying circumventions and closing loopholes. BMJ open. 2019;9(9):e028506.

6. Guillaumier A, Bonevski B, Paul C, Durkin S, D'Este C. Socioeconomically disadvantaged smokers' ratings of plain and branded cigarette packaging: an experimental study. BMJ open. 2014;4(2):e004078.

7. Maynard OM, Leonards U, Attwood AS, Bauld L, Hogarth L, Munafò MR. Effects of first exposure to plain cigarette packaging on smoking behaviour and attitudes: a randomised controlled study. BMC Public Health. 2015;15(1):240.

8. Moodie C, Mackintosh AM, Hastings G, Ford A. Young adult smokers' perceptions of plain packaging: a pilot naturalistic study. Tobacco Control. 2011;20(5):367-73. 
9. Allen F, Schwartz M. The effect of stimulation of the senses of vision, hearing, taste, and smell upon the sensibility of the organs of vision. The Journal of general physiology. 1940;24(1):105.

10. Spence C. Multisensory flavor perception. Cell. 2015;161(1):24-35.

11. Korsmeyer C. Making sense of taste: Food and philosophy: Cornell University Press; 2002.

12. Sharpe O, Conner MT, Freeman RP, Booth DA. Insight into sight, touch, taste and smell by multiple discriminations from norm. Seeing and Perceiving. 2011;24(5):485-511.

13. Krishna A, Morrin M. Does touch affect taste? The perceptual transfer of product container haptic cues. Journal of Consumer Research. 2008;34(6):807-18.

14. Moir H. Some observations on the appreciation of flavour in foodstuffs. Journal of the Society of Chemical Industry. 1936;55(8):145-8.

15. Todrank J, Bartoshuk LM. A taste illusion: taste sensation localized by touch. Physiology \& behavior. 1991;50(5):1027-31.

16. BinDhim NF, McGeechan K, Alanazi AK, Alanazi HM, Alanazi SA, Al-Hadlaq SM, et al. Evaluating the pictorial warnings on tobacco products in Arabian Gulf countries against other international pictorial warnings. Tobacco Control. 2018;27(3):261-5.

17. Sharik Association for Health Research (SharikHealth). 2015 [cited 2020 Jan]. Available from: https://sharikhealth.com/.

18. American Lung Association. Measuring Your Peak Flow Rate. Available from:

https://www.lung.org/lung-health-and-diseases/lung-disease-lookup/asthma/living-withasthma/managing-asthma/measuring-your-peak-flow-rate.html.

19. Maxwell SE, Delaney HD, Kelley K. Designing experiments and analyzing data: A model comparison perspective: Routledge; 2017.

20. Davis CS. Statistical methods for the analysis of repeated measurements: Springer Science \& Business Media; 2002.

21. Saudi Gazette. New cigarette packs free of sawdust, SFDA confirm 2019 [cited 2020 Jan]. Available from: http://saudigazette.com.sa/article/583828.

22. Arab News. Saudi Arabia warns firms over cigarette quality 2020 [cited 2020 Jan]. Available from: https://www.arabnews.com/node/1608481/saudi-arabia.

23. Sebrie EM, Barnoya J, Pérez-Stable EJ, Glantz SA. Tobacco industry successfully prevented tobacco control legislation in Argentina. Tobacco Control. 2005;14(5):e2-e.

24. Hiilamo H. Tobacco industry strategy to undermine tobacco control in Finland. Tobacco control. 2003;12(4):414-23.

\section{Figures}




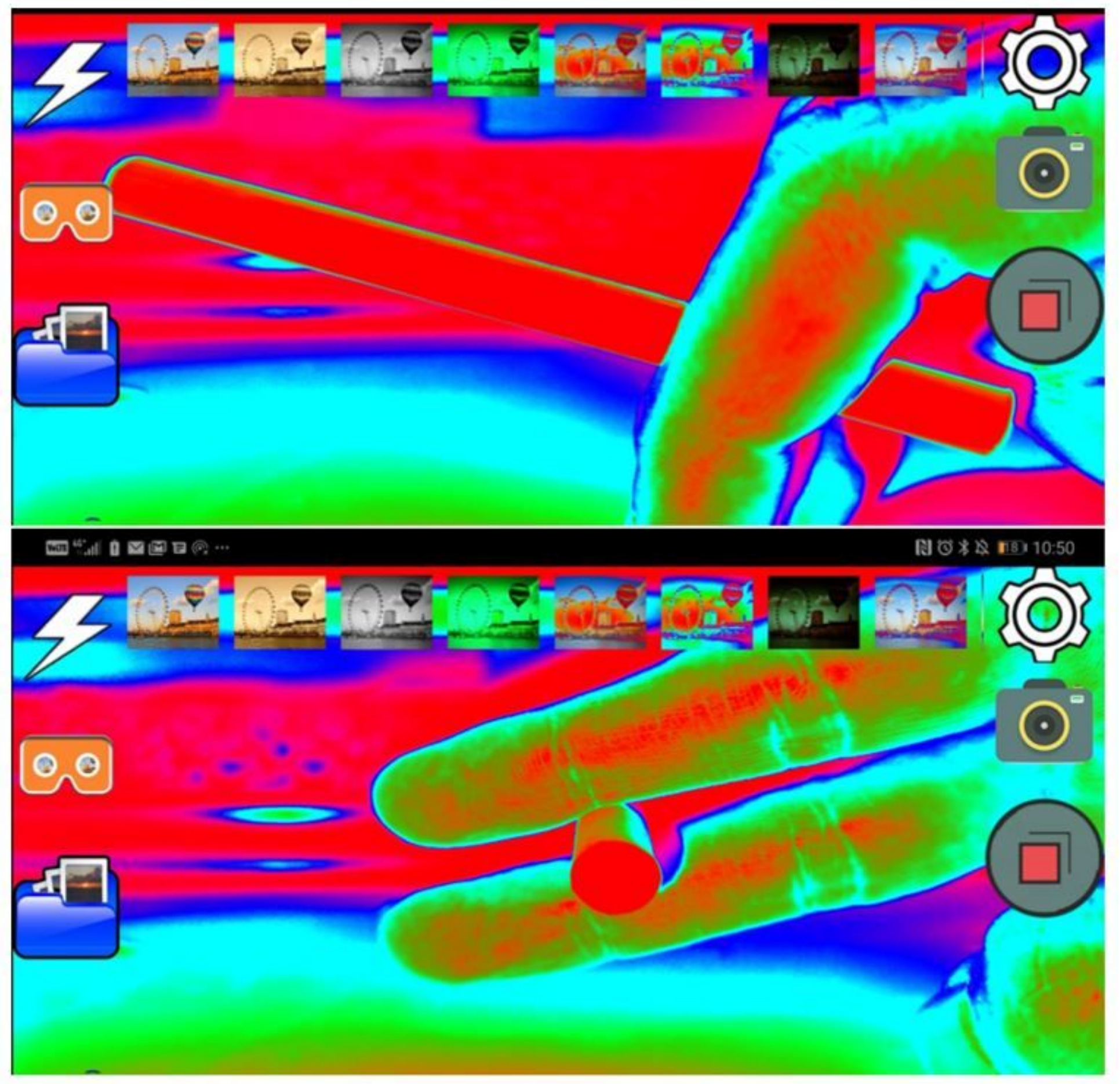

\section{Figure 1}

showed a screenshot of the cigarettes blinding app for the VR. 